\title{
DNA methylation epigenotypes in breast cancer molecular subtypes
}

\author{
Naiara G Bediaga', Amelia Acha-Sagredo², Isabel Guerra ${ }^{3}$, Amparo Viguri ${ }^{3}$, Carmen Albaina ${ }^{3}$, Irune Ruiz Diaz ${ }^{4}$, \\ Ricardo Rezola ${ }^{5}$, María Jesus Alberdi ${ }^{5}$, Joaquín Dopazo ${ }^{6}$, David Montaner ${ }^{6}$, Mertxe de Renobales ${ }^{7}$, \\ Agustín F Fernández ${ }^{8}$, John K Field ${ }^{9}$, Mario F Fraga ${ }^{10}$, Triantafillos Liloglou ${ }^{9}$, Marian M de Pancorbo ${ }^{1 *}$
}

\begin{abstract}
Introduction: Identification of gene expression-based breast cancer subtypes is considered a critical means of prognostication. Genetic mutations along with epigenetic alterations contribute to gene-expression changes occurring in breast cancer. So far, these epigenetic contributions to sporadic breast cancer subtypes have not been well characterized, and only a limited understanding exists of the epigenetic mechanisms affected in those particular breast cancer subtypes. The present study was undertaken to dissect the breast cancer methylome and to deliver specific epigenotypes associated with particular breast cancer subtypes.
\end{abstract}

Methods: By using a microarray approach, we analyzed DNA methylation in regulatory regions of 806 cancerrelated genes in 28 breast cancer paired samples. We subsequently performed substantial technical and biologic validation by pyrosequencing, investigating the top qualifying $19 \mathrm{CpG}$ regions in independent cohorts encompassing 47 basal-like, 44 ERBB2+ overexpressing, 48 luminal A, and 48 luminal B paired breast cancer/ adjacent tissues. With the all-subset selection method, we identified the most subtype-predictive methylation profiles in multivariable logistic regression analysis.

Results: The approach efficiently recognized 15 individual $\mathrm{CpG}$ loci differentially methylated in breast cancer tumor subtypes. We further identified novel subtype-specific epigenotypes that clearly demonstrate the differences in the methylation profiles of basal-like and human epidermal growth factor 2 (HER2)-overexpressing tumors.

Conclusions: Our results provide evidence that well-defined DNA methylation profiles enable breast cancer subtype prediction and support the utilization of this biomarker for prognostication and therapeutic stratification of patients with breast cancer.

\section{Introduction}

Breast cancer is a heterogeneous disease, both biologically and clinically. The molecular background behind breast cancer progression is not well understood, but is associated with the accumulation of genetic aberrations, leading to widespread gene-expression changes in breast tumor cells. Consistent with this is the presence of at least four major breast cancer subtypes with distinct expression patterns and clinical outcomes. These subtypes are termed basal-like, ERBB2+, luminal B, and luminal A [1,2]. Basal-like and ERBB2+ subtypes are

\footnotetext{
* Correspondence: marianpancorbo@gmail.com

'BIOMICs Research Group, Centro de Investigacion y Estudios Avanzados 'Lucio Lascaray', University of the Basque Country UPV/EHU, Miguel de Unamuno 3,1006, Vitoria-Gazteiz, Spain

Full list of author information is available at the end of the article
}

hormone-receptor negative and have poor prognoses. In contrast, luminal breast cancers are characterized by the expression of ER-associated genes, with luminal B tumors having poorer outcomes than luminal A tumors. Although this gene expression-based approach has proven to add significant prognostic and predictive value to pathologic staging, histologic grade, and standard clinical molecular markers [3], the high cost of expression profiling and the molecular instability of the mRNA transcripts has limited its incorporation into clinical settings, and therefore, expression-based breast cancer classification has not become a standardized method in the general practice [4]. Thus, although breast cancer stratification by gene expression is still considered the gold standard, an urgent need exists for well-defined

\section{Ciomed Central}


biomarker panels allowing breast cancer subtyping in clinical diagnostics.

Epigenetic alterations such as aberrations in DNA methylation, microRNA patterns, and post-translational modifications of histones are common molecular abnormalities in cancer [5]. Furthermore, many studies suggest that epigenetic changes are involved in the earliest phases of tumorigenesis, and that they may predispose stem/progenitor cells to subsequent genetic and epigenetic changes involved in tumor promotion [6]. Cancer-related disruption of the DNA methylome involves global genomic hypomethylation and regional hypermethylation of cytosine-phosphate-guanine (CpG) islands. The first can lead to chromosomal instability [7], whereas the second is frequently associated with promoters of tumor-suppressor genes, resulting in their transcriptional silencing. Both such alterations have been frequently observed in breast cancer [8-19].

Analogous to transcriptomic profiling, DNA methylation profiling is considered to allow the molecular classification of human malignancies and monitoring cancer progression based on a tumor-specific methylation signature [20]. At the same time, it facilitates biomarker discovery for the clinical implementation of this process.

Previous epigenetic analyses have identified aberrant DNA methylation signatures associated with molecular subtypes of breast cancer through hormone-receptor and human epidermal growth factor 2 (HER2) status [21-23]; however, very limited information is available on global methylation changes associated with each molecular subtype, as previous studies focused on individual candidate tumor-suppressor genes by using locusspecific methods. Here we have applied an array-based method [24] for comprehensive DNA methylation profiling to identify differentially methylated genes in breast cancer molecular subtypes. This approach efficiently recognized 15 differentially DNA-methylated loci, which were further validated through pyrosequencing in an independent cohort encompassing 47 basal-like, 44 ERBB2+ overexpressing, 48 luminal A, and 48 luminal B paired breast cancer/adjacent tissues. Our results provide strong evidence for the existence of tumor subtypespecific aberrant methylation profiles, which might be inducers of some transcriptional changes taking place in breast cancer molecular subtypes.

\section{Materials and methods}

\section{Patients and tumor characteristics}

Samples and associated clinicopathologic data were obtained from the Anatomy Pathological Services of the Txagorritxu Hospital, Oncologic Institute of Donostia, and Donostia Hospital (Basque Country). Samples of breast tumor and corresponding adjacent normal-appearing tissue (located at least $2 \mathrm{~cm}$ away from the site at which the tumor was sampled) were collected from 215 patients diagnosed with a ductal infiltrative breast carcinoma.

DNA-methylation measurements were performed on DNA isolated from paraffin-embedded primary breast cancer. All breast specimens were reviewed by experienced pathologists. The inclusion criteria were the availability of the paraffin-embedded tissue, tumor size between 1 and $3 \mathrm{~cm}$, histologic grade between 1 and 3, and estrogen receptor (ER)-, progesterone receptor (PR)-, HER2-, CK5/6+, or CK14+, or EFGR+ for the basal-like tumors, ER+, PR \pm , and HER2+ for the Luminal B, ER-, PR-, and HER2+ for the ERBB2+ tumor group, and ER+, PR+, and HER2- for the Luminal A. Additional data such as Ki-67 status, p53 mutation, and nodal involvement were also registered. Ethical approval for the study was obtained from the corresponding Ethics Committees of the Institutions involved.

\section{Macrodissection, DNA extraction, and bisulfite modification}

To minimize contamination in the methylation analysis, we isolated breast cancer cells and paired normal breast epithelial cells from tissues by manual macrodissection. In brief, $10-\mu \mathrm{m}$ sections were cut from each archival formalin-fixed paraffin-embedded (FFPE) tissue block. For each pair of tissues, the presence of tumor cells in malignant tissues and the absence of cancer cells in normal tissues were confirmed by histopathologic examination.

A total of $500 \mu \mathrm{l}$ of buffer TE $\mathrm{pH} 9$ was added to each sample and heated at $100^{\circ} \mathrm{C}$ for 20 minutes by using a water bath. After heating, a cooling step of 5 minutes was allowed before adding $20 \mu \mathrm{l}$ of proteinase K. Samples were incubated at $56^{\circ} \mathrm{C}$ overnight until all the tissue fragments were completely dissolved. Subsequent extraction and purification procedures were performed after the next steps: addition of $500 \mu \mathrm{l}$ phenol/chloroform/ isopropanol alcohol $(25: 24: 1)$ to the digested tissue, followed by mixing for 10 minutes and centrifugation at $12,000 \mathrm{rpm}$ for 10 minutes. The supernatant fluid was removed to an autoclaved microtube, and one volume of chloroform/isopropanol alcohol (25:1) was added, mixed by vortexing, and centrifuged at $12,000 \mathrm{rpm}$ for 10 minutes. The upper aqueous supernatant was purified by using a DNA purification kit, following the manufacturer's instructions (Qiagen, Valencia, Spain), and the final yield of DNA was dissolved in $50 \mu \mathrm{l}$ of buffer TE. Sodium bisulfite modification of $1.5 \mu \mathrm{g}$ DNA was done with the EZ DNA methylation kit (Zymo Research, Orange, CA, USA) by following the manufacturer's protocol. 


\section{Marker discovery study Illumina GoldenGate Methylation Cancer Panel 1}

The Illumina GoldenGate Methylation Cancer Panel was used to analyze $550 \mathrm{ng}$ of starting bisulfite-modified genomic DNA. Methylation was represented as a continuous value from 0 (completely unmethylated) to 1 (completely methylated). This value is calculated by subtracting background hybridization levels obtained from negative control probes on the array and calculating the ratio of the fluorescent signal from the methylated allele (M) to the sum of the fluorescent signals from both unmethylated $(\mathrm{U})$ and methylated alleles $(|\mathrm{U}|+|\mathrm{M}|+100)$.

\section{Differential methylation analysis}

Microarray data were analyzed to identify the most significant tumor subtype-specific changes relative to the adjacent tissue. Differential methylation was assessed by comparing the mean methylation level (b-value) of samples with the mean b-value of the corresponding adjacent tissue by using BeadStudio (San Diego, CA, USA) and Qlucore Omics Explorer 2.0 (Qlucore AB, Lund, Sweden) software. Selection of the most significantly differentially methylated loci in each tumor subtype was based on (a) $\Delta b$ value difference of at least 0.20 between the tumors and reference group; (b) an FRD-corrected $P$ value cut off of $P<0.001$, as determined by a twotailed $t$ test [25]; and (c) a $P$ value $<0.05$ when comparing mean methylation values among the studied tumor subtypes by using analysis of variance (ANOVA) test with Benjamini-Hochberg FDR multiple testing correction. The methylation data have been deposited in NCBI's Gene Expression Omnibus (GEO) [26] and are accessible through GEO Series accession number [GEO: GSE22135].

\section{Validation of the data by pyrosequencing $P C R$ and pyrosequencing reactions}

Selected markers from microarray data analysis were further validated in a larger sample size by bisulfite/pyrosequencing. Additionally, four candidates from the literature were included: $L I N E-1$, to measure the global hypomethylation of the tumors [27], and Let-7a, Mir$10 a$, and Mir-93 microRNAs, because they have been reported to be differently expressed in breast cancer molecular subtypes [28], and their expression might be regulated by methylation in some tumor subtypes, and thus provide new potential subtype-specific biomarkers.

For the methylation analysis, $1.5 \mu \mathrm{g}$ of genomic DNA was treated with sodium bisulfite, by using the EZ DNA methylation Kit (Zymo Research, Orange, CA, USA) according to the manufacturer's protocol. All primers were designed by using the Assay Design Software (Biotage, Uppsala, Sweden) and synthesized by MWG (Ebersberg, Germany). PCR amplifications were performed by using Qiagen HotStarTaq Master Mix Kit
(Qiagen, Valencia, Spain), 7.5 $\mu M$ biotinylated primer, $15 \mu M$ nonbiotinylated primer, and $2 \mu \mathrm{l}$ of bisulfitetreated DNA (60 ng). PCR primer sequences, PCR conditions, and sequencing primer sequences are given in Table $\mathrm{S} 1$ in Additional file 1 . The quality and quantity of the PCR product was confirmed by agarose gel (2\%) electrophoresis before the cleanup and pyrosequencing analysis. Pyrosequencing was carried out by using the SQA kit (Biotage, Uppsala, Sweden) on a PSQ 96MA Pyrosequencer (Biotage), and the methylation index was calculated by using the Pyro Q-CpG software (Biotage).

\section{Validation data analysis}

Methylation status in tumor versus adjacent tissue

Methylation status was assessed at the studied markers, as previously described by Feng et al. [21]. Taking advantage of paired normal/tumor samples, normal tissues' value was considered as the reference. If using the pooled normal samples' mean plus twice the standard deviation as a cut-off point (minimum, 10\%), we estimated the probability of the methylation level for a normal-appearing tissue being lower than the cut-off point is $<96 \%$. Thus, it is reasonable to assume that samples with a methylation value larger than the cut-off point are likely to be abnormal (or positive). A paired $t$ test was used to determine whether a statistically significant change was present in the methylation of the markers examined between the tumors and adjacent tissues. Additionally, to allow the assessment of the observed methylation at multiple promoters as a continuous variable, Z-score analysis was used [29,30]. A Z score for each gene was calculated by using the given formula:

Mean of CpG methylation density of the assessed promoter for each sample - Mean of methylation density for the tumor panel)/SD of methylation density.

A mean $\mathrm{Z}$ score was calculated by integrating the promoter-specific Z scores and used as a simple score characterizing mean methylation density. In this analysis, a $\mathrm{Z}$ score $>0$ means methylation greater than the population mean.

\section{Comparison between methylation status, breast can-} cer molecular subtypes, and clinicopathologic characteristic A one-sample Kolmogorov-Smirnov test was used to evaluate fitness to normal distribution of continuous parameters. Differences in promoter methylation among tumor subtypes were analyzed with ANOVA or KruskalWallis tests as appropriate. The Wilcoxon signed-rank test was used to compare methylation in paired samples. If differences between two independent groups or clinicopathologic characteristics had to be considered, a parametric test (Student $t$ test) or nonparametric test (Mann-Whitney $U$ test) was used. Comparisons of categoric variables were made by using Fisher's Exact and Pearson's $\chi^{2}$ tests. All reported $P$ values are two-tailed and considered statistically significant if $P<0.05$. 
Subtype classification Multivariate logistic regression (MLR) analysis was performed on those biomarkers showing significance in univariate analysis to identify potential biomarker panels capable of discriminating breast cancer subtypes with the best sensitivity and specificity. Models including all possible combinations were constructed and tested by Mallows' $\mathrm{Cp}$ selection criterion. The false discovery rate (FDR) of classifying breast cancer subtypes was determined in the best models, and we selected those significant at the FDR $<0.2$ level.

Supervised hierarchic clustering based on genes selected in the models was performed by using an ANOVA test (Benjamini-Hochberg FDR multiple testing corrected [25]) to confirm results obtained by MLR (Qlucore Omics Explorer 2.0; Qlucore AB, Lund, Sweden). DNA methylation profiles were standardized to have a mean of zero and a standard deviation of 1 , and clustering was performed by using the euclidean method and average linkage.

\section{Ethical considerations}

The present study involved analysis of DNA from archival tissue with no subject intervention. No identities were linked to subject records. This study was approved by the Txagorritxu Hospital Review Board under the category of exempt status, and no consent form was required from the participants.

\section{Results}

Breast cancer subtypes display distinct clinicopathologic characteristics

Patient characteristics are presented in Table 1. Basallike and ERBB2+ subtypes were more aggressive tumors than luminal A and B ones, as indicated by their histologic and Ki-67 grades $(P<0.01)$, but the basal-like subtype showed less nodal involvement when compared with other subtypes $(P<0.01)$. Additionally, p53 mutation was significantly less frequent in the luminal A subgroup $(P<0.05)$

\section{Array-based DNA methylation-profiling approach identifies some breast cancer subtype-specific methylation changes}

DNA methylation-profiling analysis at 1,505 CpG loci was performed in 30 paired breast cancer/adjacent tissues representing the four major breast cancer subtypes. Two of the tumor samples were eliminated from further analysis secondary to their low methylation levels. The final cohort consisted of 60 samples: 28 paired breast cancer/ adjacent tissues and four samples from the peritumoral region. Extensive DNA methylation changes between tumor and adjacent tissues were revealed after supervised clustering of the samples (Figure 1; Table S2 in Additional file 2). To identify CpGs with highly significant tumor subtype-specific changes, stringent cut-offs $(P<0.001$ and
$\Delta \mathrm{b}>0.20$ ) were set for methylation-level changes relative to controls for each tumor subtype. Distinct CpG loci groups were identified, showing differential methylation profiles in tumor subtypes. We focused in those genes that were significantly differently methylated in the basal-like tumor subtype (HOXA9, SOX1, VAMP8, and TNFRS10D) and those that were significantly hypermethylated in the luminal B tumors (NPY, RASSF1, HS3ST2, DBC1, FGF2, CD40. SPARC, JAK3, SOX17, PRKCDBP, and TAL1). Additionally, we compared mean methylation values among the studied tumor subtypes by using ANOVA and observed that NPY, PRKCDBP, and RASSF1 showed lower methylation levels in the basal-like tumor subgroup when compared with other subtypes, and that DBC1, HS3ST2, $F G F 2$, and $C D 40$ displayed higher hypermethylation levels in the luminal B subtype than in the other subgroups.

The microarray was technically validated by means of pyrosequencing. Comparison of quantitative methylation values at $5 \mathrm{CpG}$ loci by both GoldenGate array and pyrosequencing in 20 samples confirmed the accuracy of the array-based measurements (mean $r^{2}=0.75$; range, 0.56 to 0.91; Table S3 in Additional file 3).

\section{Validation of differential methylation events in a large panel of breast cancer tissue samples}

For the validation study, pyrosequencing assays for 19 CpG loci were conducted in an independent cohort of 187 normal/breast cancer paired samples encompassing 47 basal-like, 44 ERBB2+, 48 luminal $\mathrm{A}$, and 48 luminal B samples. Differential methylation between tumor and adjacent tissue was confirmed in the validation panel (Figure 2). Among the loci displaying $>95 \%$ specificity (that is, very low DNA methylation frequency in normal tissue), the frequency of hypermethylation in tumor tissue ranged from $28 \%$ ( $D B C 1$, deleted in bladder cancer 1) to $75 \%$ (JAK3, Janus kinase 3), (see Table S4 in Additional file 4). Besides, VAMP8 (vesicle-associated membrane protein 8) was hypomethylated in $82 \%$ of the tumors, and LINE-1, the global methylation marker, was highly methylated in all normal tissues (mean $=68 \% \pm 1.9 \%$ ), whereas a statistically significant decrease was observed in tumors (mean $=63 \% \pm 5.4 \% ; P<0.001$ ).

Further to define those CpG loci differentially methylated in breast cancer subtypes, data were filtered on significance of differences by using the Bonferronicorrected ANOVA test $(P<0.05)$. We found that 15 of the 19 genes involved displayed different methylation profiles in analyzed breast tumor subtypes (Table 2). LINE-1, HOXA9, TAL1, and SPARC were excluded, as they showed no significant difference. During the validation study, we confirmed that promoter methylation of NPY, RASSF1, TNFRS10D, PRKCDBP, Let-7a, and $V A M P 8$ genes was lower in the basal-like subtype when 
Table 1 Patient characteristics of both marker discovery and validation studies

\begin{tabular}{|c|c|c|c|c|c|}
\hline & & \multicolumn{4}{|c|}{ Type } \\
\hline & & Basal & ERBB2+ & Luminal B & Luminal A \\
\hline Number of patients & & 59 & 49 & 54 & 53 \\
\hline \multicolumn{6}{|l|}{ Age (years) } \\
\hline & Mean & 56.1 & 57.1 & 56.9 & 62.9 \\
\hline & Median & 53 & 57.5 & 51 & 61 \\
\hline & Range $(95 \% \mathrm{Cl})$ & $51.5-60.7$ & $52.5-61.6$ & $51.8-61.7$ & $56.9-68.7$ \\
\hline \multicolumn{6}{|l|}{ Tumor size $(\mathrm{cm})$} \\
\hline & Mean & 2.4 & 2.8 & 3.2 & 3.1 \\
\hline & Range $(95 \% \mathrm{Cl})$ & $2.1-2.7$ & $2.1-3.5$ & $2.5-3.5$ & $2.4-3.7$ \\
\hline \multicolumn{6}{|l|}{ Histologic grade (\%) } \\
\hline & 1 & 5.0 & 0.0 & 0.0 & 6.0 \\
\hline & $\|$ & 47.1 & 24 & 40.0 & 55.5 \\
\hline & III & 48.9 & 76 & 60.0 & 38.5 \\
\hline \multicolumn{6}{|c|}{ Nodal involvement (\%) } \\
\hline & Positive & 35.5 & 63.4 & 72.2 & 57.3 \\
\hline & Negative & 64.4 & 36.6 & 27.8 & 42.7 \\
\hline \multicolumn{6}{|c|}{ Ki-67 overexpression (\%) } \\
\hline & $<10 \%$ & 0.0 & 8.9 & 21.9 & 27.8 \\
\hline & $10 \%-25 \%$ & 0.0 & 36.1 & 31.2 & 66.7 \\
\hline & $>25 \%$ & 100.0 & 56.0 & 46.9 & 5.6 \\
\hline \multicolumn{6}{|l|}{ p53 mutation (\%) } \\
\hline & Positive & 47.2 & 44.1 & 21.8 & 14.8 \\
\hline & Negative & 52.8 & 55.9 & 88.2 & 85.2 \\
\hline
\end{tabular}

compared with HER2-overexpressing tumors (luminal B and ERBB2+). SOX1 and SOX17 showed different methylation levels between luminal $B$ and luminal A tumors. We also observed that HS3ST2, DBC1, FGF2, CD40, JAK3, Mir-93, and Mir-10a markers displayed higher methylation levels in luminal B and ERBB2+ subtypes than in the basal-like and the luminal A tumors. Consequently, the validation study confirmed that these markers were suitable for development of tumor-subtype methylation profiles.

One of the most interesting aspects of the validation study was to observe that whereas the microarray analysis showed no specific methylation profile for HER2overexpressing tumors (luminal B and ERBB2+ tumors), the validation study (carried out in a larger sample size) demonstrated that HER2-overexpressing tumors had a common methylation profile.

The analysis of DNA-methylation profiles with regard to the tumors' clinicopathologic features revealed that hypermethylation of HS3ST2 was more prevalent in patients with nodal involvement $\left(\chi^{2}=10.61 ; P<0.001\right)$, whereas RASSF1 hypermethylation was associated with the presence of p53 mutations $\left(\chi^{2}=7.51 ; P<0.001\right)$ and high Ki-67 index $\left(\chi^{2}=15.63 ; P<0.001\right)$. In addition, promoter hypermethylation of TAL1 and SPARC genes was more frequent in tumors with high histologic grade $\left(\chi^{2}=6.41\right.$ and $\chi^{2}=6.21$, respectively; $\left.P<0.05\right)$.

We next combined all the methylation data into a single variable, the mean $\mathrm{Z}$ score, to allow further analysis. As shown in Figure 2c, mean methylation density in tumors was significantly higher than that in adjacent tissue $[-1.26(\mathrm{CI},-1.26$ to -1.17$)$ and $-0.01(\mathrm{CI},-0.81$ to $0.72) ; P<0.001]$. Additionally, we found significant differences in the mean $\mathrm{Z}$ scores among the different breast cancer subtypes [basal-like, -0.45 (-0.58 to -0.32); luminal B, 0.24 (0.09 to 0.39); ERBB2+, 0.22 (0.05 to 0.41); and luminal A, $-0.02(-0.14$ to 0.09$) ; P<0.001$ ] (Figure 3c), and that significantly higher Z-score epigenotypes were related to LINE-1 hypomethylated tumors [0.27 (CI, 0.04 to 0.28 ) and -0.06 (CI, -0.13 to $0.02 ; P<0.05$ ], HER2overexpressing breast cancers [0.25 (CI, 0.15 to 0.37 ) and -0.22 (CI, -0.33 to -0.22 ); $P<0.001]$, and node-positive tumors $[0.11(\mathrm{CI},-0.01$ to 0.19$)$ and $-0.10(\mathrm{CI},-0.25$ to $0.4) ; P<0.05]$, which are features associated with clinically more-aggressive tumors. Paradoxically, highly proliferative and p53-mutated tumors, both associated with the basal-like phenotype, showed significantly lower $\mathrm{Z}$ scores of methylation [-0.17 (CI, -0.35 to 0.01 ) and 0.11 (CI, -0.03 to 0.26$) ; P<0.001]$ and $[-0.23(\mathrm{CI},-0.44$ to $-0.11)$ and 0.12 (CI, -0.02 to 0.26$) ; P<0.001]$ respectively. 


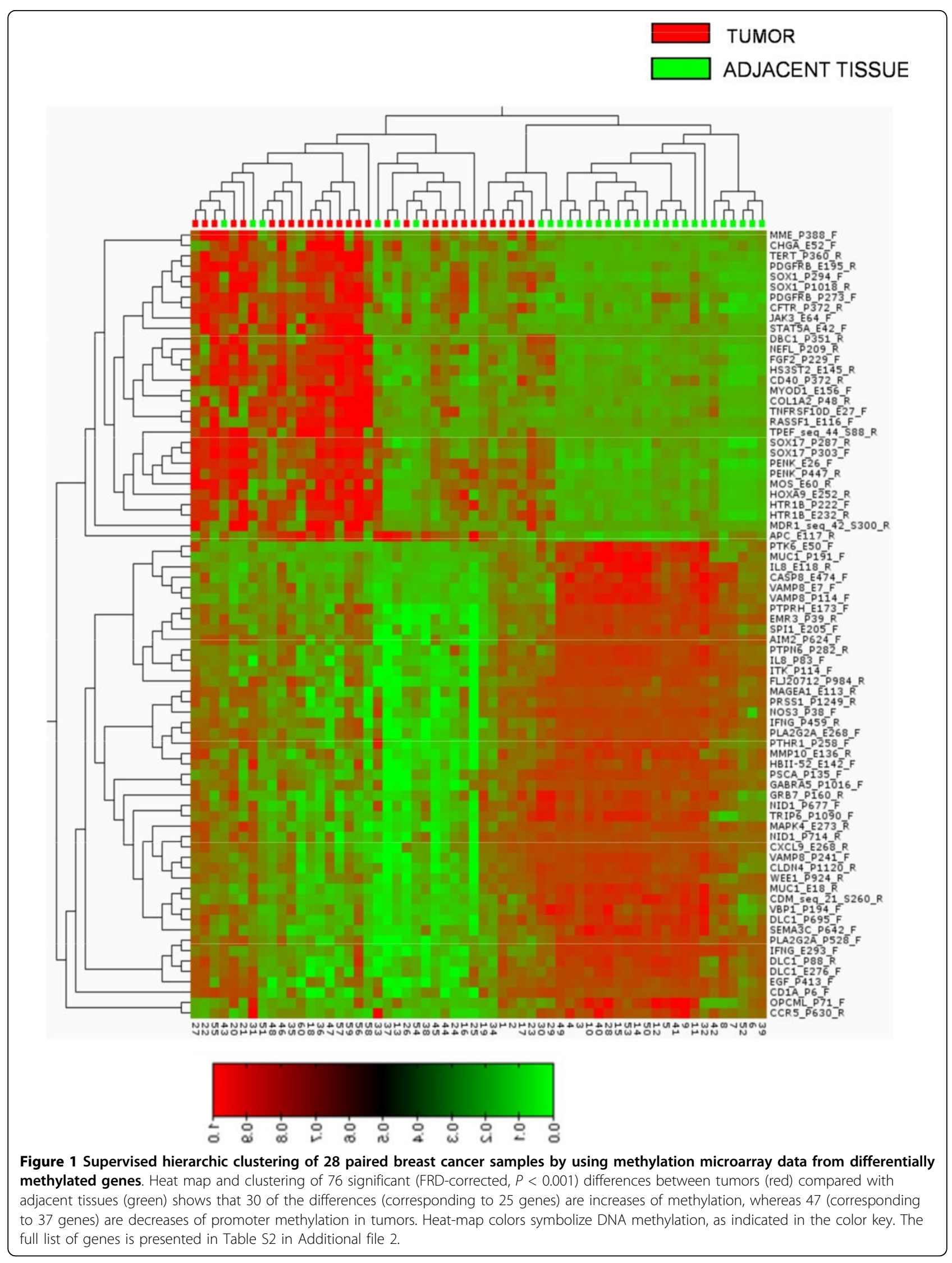


(a)

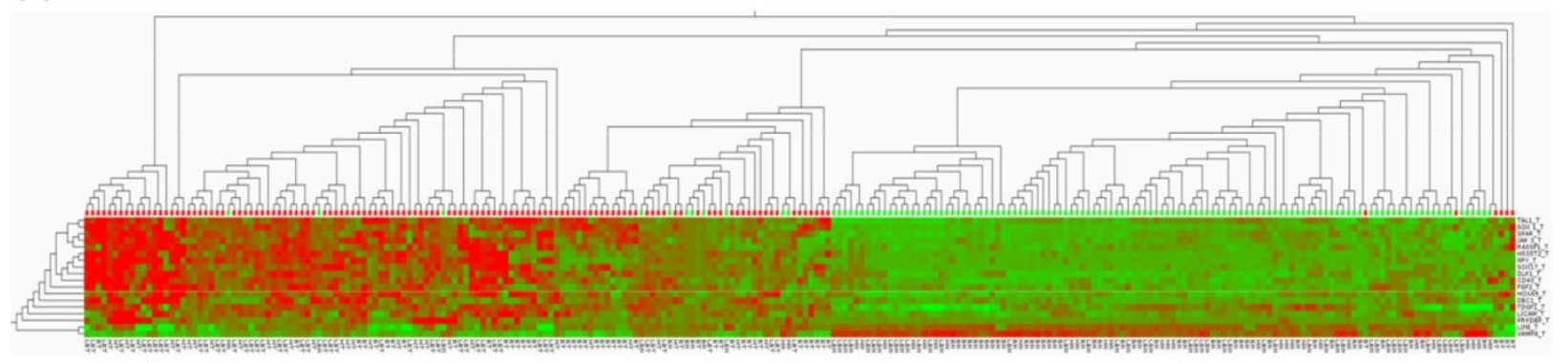

(b)

(c)
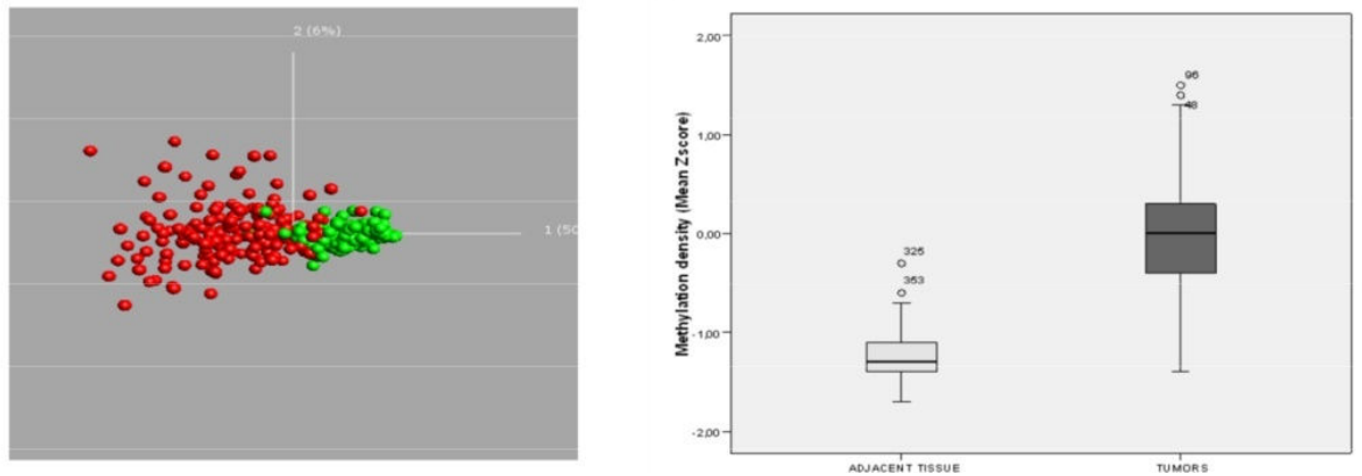

Figure 2 Supervised hierarchic clustering of 187 paired breast cancer samples by using methylation data from selected genes in the validation study. (a) Heat map and clustering of significant (FRD-corrected, $P<0.01$ ) differences between tumors (red) and adjacent tissues (green). Heat-map colors symbolize DNA methylation, as indicated in the color key. (b) Principal component analysis (PCA) plots showing separation between tumor and adjacent tissue. (c) Box plots showing lower methylation density in adjacent tissue compared with tumors.

\section{Novel DNA methylation profiles with subtype-predictive value}

Selected loci were further processed to identify the best biomarker panel allowing classification in tumor subtypes. Multivariate logistic regression (MLR) analysis was performed to identify potential biomarker panels with the best sensitivity, specificity, and false discovery rate (FDR). We first pruned all the variables showing significance in univariate analysis (Table 3) by using all-subset selection with Mallows' Cp optimization criteria, and recognized biomarker panels capable of discriminating basal-like and HER2-overexpressing subtypes at FDR 0.2 level. Specifically, basal-like tumors were inversely associated with hypermethylation of NPY, FGF2, HS3ST2, RASSF1, and Let-7a markers (sensitivity, 75\%; specificity, 95\%; and FDR, 0.17), whereas HER2-overexpressing subtypes were strongly correlated to hypermethylation of NPY, FGF2, HS3ST2, RASSF1, and Let-7a (sensitivity, 74\%; specificity, $80 \%$, and FDR, 0.18).

The supervised hierarchic-clustering analysis based on these five genes resulted in three different clusters of tumor groups (C1 to C3) (Figure 3). Samples fitting in cluster $\mathrm{C} 1$ (characterized by lack of methylation) were mostly basal-like tumors; cluster $\mathrm{C} 2$ was the most heterogeneous one with regard to both methylation status of the genes and samples fitting into it. This cluster contains the majority of the luminal A samples, but also includes some luminal B and ERBB2+ tumors. Conversely, cluster C3 (hypermethylated in almost all the analyzed markers) was basically composed of luminal $\mathrm{B}$ and ERBB2+ samples (HER2-overexpressing subtypes).

\section{Discussion}

Identification of gene expression-based breast cancer subtypes is considered a critical means of prognostication, and furthermore, an important predictive marker for the response to treatment with endocrine therapy. However, analytic tests relying on RNA measures are difficult to standardize and implement because of the instability of the mRNA transcripts. DNA-methylation profiles reflect phenotypically important differences in gene transcription and, in contrast to most mRNAs, a very stable structure, making DNA-methylation profilebased diagnostic tests highly accurate and reproducible [31]. By use of two independent cohorts of invasive 
Table 2 Methylation levels in breast cancer subtypes

\begin{tabular}{|c|c|c|c|c|c|}
\hline \multirow[t]{2}{*}{ Gene } & \multicolumn{5}{|c|}{ Methylation level (mean \pm SD) (positive rate ${ }^{a}$ ) } \\
\hline & Basal like & Luminal B & ERBB2+ & Luminal $A$ & $P$ value \\
\hline CD40 & $24.3 \pm 12.3(21.7)$ & $30.8 \pm 11.9(36.2)$ & $30.8 \pm 11.9(33.4)$ & $27.4 \pm 7.9(23.9)$ & $<0.001$ \\
\hline$D B C 1$ & $12.8 \pm 9.2(15.9)$ & $19.9 \pm 10.3(34.1)$ & $17.8 \pm 8.2(42.7)$ & $14.0 \pm 7.3(19.5)$ & $<0.001$ \\
\hline FGF2 & $7.4 \pm 7.2(11.1)$ & $23.1 \pm 11.2(63.8)$ & $32.2 \pm 13.9(67.5)$ & $15.0 \pm 13.4(30.8)$ & $<0.001$ \\
\hline HOXA9 & $21.1 \pm 12.0(61.8)$ & $22.5 \pm 9.4(64.7)$ & $28.4 \pm 10.3(83.8)$ & $20.3 \pm 8.0(78.1)$ & NS \\
\hline HS3ST2 & $10.3 \pm 11.9(31.0)$ & $23.9 \pm 12.9(75.0)$ & $26.8 \pm 12.3(79.8)$ & $17.5 \pm 13.2(40.4)$ & $<0.001$ \\
\hline JAK 3 & $28.0 \pm 15.3(53.5)$ & $36.8 \pm 15.2(87.5)$ & $33.3 \pm 13.7(74.1)$ & $28.8 \pm 10.3(83.0)$ & $<0.001$ \\
\hline Let-7a & $33.9 \pm 11.3(19.1)$ & $55.1 \pm 10.9(72.9)$ & $48.8 \pm 15.7(65.7)$ & $53.5 \pm 8.4(80.0)$ & $<0.001$ \\
\hline LINE-1 & $63.8 \pm 3.2(30.2)$ & $63.1 \pm 6.2(40.4)$ & $63.8 \pm 7.8(40.2)$ & $62.8 \pm 4.7(30.4)$ & NS \\
\hline Mir-10a & $15.5 \pm 4.2(36.4)$ & $25.2 \pm 5.8(70.8)$ & $23.1 \pm 10.1(77.4)$ & $20.8 \pm 11.3(63.2)$ & $<0.001$ \\
\hline Mir-93 a & $55.6 \pm 7.3(23.9)$ & $60.6 \pm 6.5(57.8)$ & $61.2 \pm 8.9(62.1)$ & $61.3 \pm 6.5(47.5)$ & $<0.001$ \\
\hline NPY & $14.9 \pm 4.9(35.6)$ & $38.9 \pm 14.6(95.8)$ & $34.5 \pm 14.5(80.9)$ & $27.4 \pm 14.8(78.6)$ & $<0.001$ \\
\hline PRKCDBP & $7.6 \pm 6.1(4.3)$ & $13.2 \pm 10.0(12.5)$ & $15.6 \pm 10.1$ & $10.6 \pm 8.4(15.0)$ & $<0.001$ \\
\hline RASSF1 & $20.4 \pm 11.9(35.7)$ & $33.9 \pm 12.9(79.2)$ & $35.6 \pm 10.8(74.5)$ & $23.5 \pm 5.9(73.9)$ & $<0.001$ \\
\hline sox 1 & $19.8 \pm 10.7(75.0)$ & $24.5 \pm 11.2(81.3)$ & $21.5 \pm 10.1$ (78.6) & $20.7 \pm 10.1$ & $<0.001$ \\
\hline SOX17 & $19.1 \pm 15.6(59.1)$ & $22.4 \pm 14.2(70.8)$ & $23.2 \pm 14.7(62.4)$ & $14.1 \pm 7.2(32.4)$ & $<0.001$ \\
\hline SPARC & $35.3 \pm 10.7(48.9)$ & $33.7 \pm 10.2(60.4)$ & $34.5 \pm 12.2(67.3)$ & $31.1 \pm 11.0(68.1)$ & NS \\
\hline TAL1 & $38.8 \pm 12.5(59.1)$ & $33.9 \pm 11.3(56.5)$ & $37.7 \pm 11.6(60.4)$ & $27.4 \pm 7.9(52.3)$ & NS \\
\hline TNFRS1OD & $12.5 \pm 12.6(27.8)$ & $18.9 \pm 8.8(60.9)$ & $23.5 \pm 11.3(65.7)$ & $19.6 \pm 9.2(60.0)$ & $<0.001$ \\
\hline VAMP8 & $8.8 \pm 3.8(93.1)$ & $12.4 \pm 4.4(72.1)$ & $11.4 \pm 3.9(77.2)$ & $11.9 \pm 4.1(79.2)$ & $<0.001$ \\
\hline
\end{tabular}

${ }^{\text {a }}$ positive rate using the sample mean plus 2 times the SD of the pooled normal samples (and minimum $10 \%$ methylation) as a cut-off point. ${ }^{b} P$ value computed by using the ANOVA or Kruskal-Wallis tests, as appropriate.

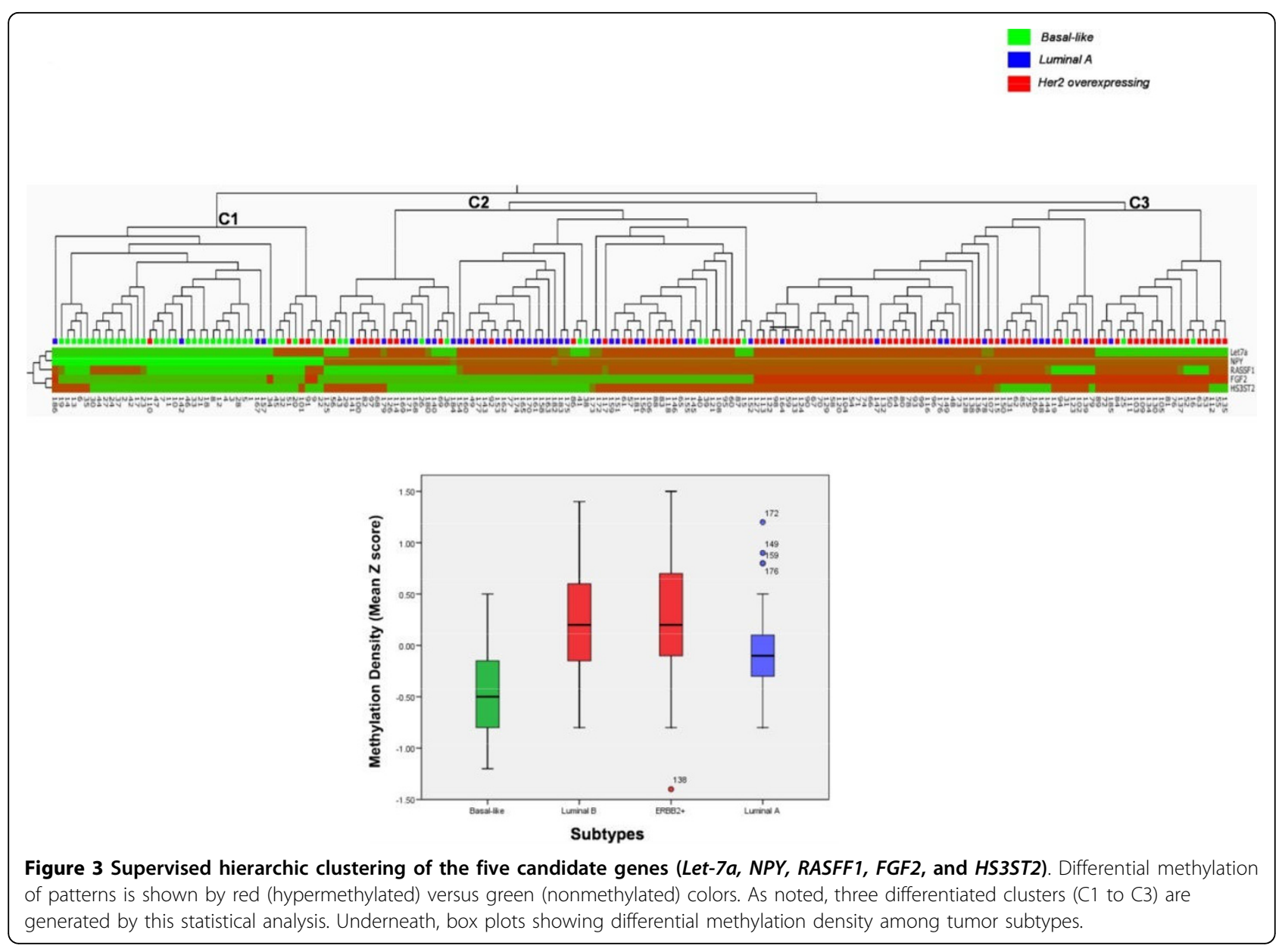


Table 3 Subtype-specific methylation profiles in univariate analysis

\begin{tabular}{ll}
\hline Tumor subtype & Related genes $^{\text {a }}$ \\
\hline Basal-like & JAK3(-), NPY(-), RASSF1(-), SPARC(-), DBC1(-), FGF2(-), HS3ST2(-), PRKCDBP(-), TNFRS10D(-), and VAMP8(+) \\
HER2 overexpressing & NPY(+), DBC1(+), FGF2(+), RASSF1(+), HS3ST2(+), SOX17(+), and TNFRS10D(+) \\
Luminal A & SOX1(-), SOX17(-), and VAMP8(+) \\
\hline
\end{tabular}

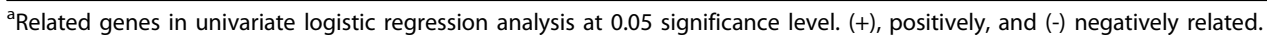

breast carcinomas, our study is, to our knowledge, one of the first to deliver specific methylation profiles associated with basal-like, ERBB2+, luminal A, and luminal $B$ molecular subtypes of breast cancer.

Statistical analysis of the methylation microarray data revealed extensive DNA-methylation changes between tumor and adjacent tissue (Figure 1, Table S2 in Additional file 2) and concurrence of some of these DNA-methylation changes with particular breast cancer molecular subtypes and tumor morphologies. Applying stringent criteria, we identified significant differential methylation among the tumor subtypes in 15 of the 1,505 screened CpG islands. Subsequent validation of the selected genes plus four more from the literature by pyrosequencing in an independent cohort of 187 breast tumor/normal pairs, confirmed that genes tested have significantly altered methylation profiles in comparison to normal-appearing adjacent tissue, and that most of the candidate genes (15 of 19) displayed significantly different methylation profiles between different tumor subtypes. These results corroborate the potential usefulness of the studied markers for the development of a methylation marker panel defining tumor subtypes. Moreover, the current study represents the first evidence for DNA hypermethylation of NPY, FGF2, CD40, TAL1, JAK3, SPARC, PRKCDBP, DBC1, SOX1, TNFRS10D, Let-7a, Mir-10a, and Mir-93 and hypomethylation of VAMP8 in breast cancer.

Several genes have been previously reported to be aberrantly methylated in breast cancer (reviewed in [32-34]). Furthermore, methylation in breast cancer has already been connected to breast cancer molecular subtypes, but these observations require further confirmation. As previously suggested, we found that basal-like, ERBB2+, luminal A, and luminal B molecular subtypes displayed specific methylation profiles. Specifically, HER2-enriched breast tumors (ERBB2+ and luminal B) were associated with the hypermethylation of several genes related to cancer development (Table 3). These results are in accordance with previous studies stating that HER2/neu breast cancers are associated with preferential hypermethylation of several genes [21-23]. In addition, Terada et al. [35] recently found that frequent CpG islands methylation is highly associated with HER2 amplification. Conversely, we observed that basal-like tumors were inversely related to promoter methylation of many of the studied genes (Table 3) and showed the lowest methylation levels among the studied subtypes, as indicated by the mean $\mathrm{Z}$-score values (Figure 3c). These findings are consistent with the recent observations made by Holm et al. [36], who reported that basal-like tumors have low methylation levels of several CpG sites, whereas luminal B tumors display high methylation levels.

Additionally, our efforts to identify and validate breast cancer subtype-specific epigenotypes resulted in a significant model based on five biomarkers, which is capable of discriminating basal-like and HER2-overexpressing subtypes. Basal-like tumors showed lack of methylation at NPY, FGF2, HS3ST2, RASSF1, and Let-7a markers, whereas HER2-overexpressing tumors (luminal B and ERBB2+ subtypes) were related to hypermethylation of these markers.

Several authors have speculated that these genomically defined tumor subtypes may represent transformation of stem cells. Some of these hypotheses suggest that mammary stem cells progress to a luminal progenitor state (with an expression pattern similar to that identified in basal breast cancer), which then progresses to differentiated cells with more luminal characteristics [37]. As can be deduced, this hypothesis locates basal-like tumors in an intermediate differentiation step between the mammary stem cells and the more-differentiated luminal subtypes, which would explain their poor prognosis despite response to chemotherapy [38]. Many studies have been conducted to define methylation profiles in human stem cells [39-42]. Likewise, Calvanesse et al. [43] compared the methylation status in human embryonic stem cells (hESCs), cancer cell lines, and normal human primary tissues by using the same platform as that in our study. Their finding was consistent with the view that genes aberrantly hypermethylated in cancer (that is, not hypermethylated in normal tissues) were not hypermethylated in hESCs, termed by these authors the classic class A cancer hypermethylated genes. Interestingly, all the CpG sites related to basal-like tumors in univariate regression analysis, with the exception of RASSF1 (Table 3), belonged to this committed class A category, and in a manner analogous to hESCs; they were not hypermethylated in basal-like tumors, nor in normal breast, but they were hypermethylated in the rest of breast cancer subtypes, suggesting that basal-like 
tumors may share some similarities in their methylation patterns with those of the hESC cell lines. These shared methylation signatures may reinforce the hypothesis of basal-like tumors arising from a mammary stem cell and progressing to differentiated cells with more luminal characteristics (HER2 enriched, luminal A and B). A recent study by Holm et al. [36] also supported this hypothesis; they observed that basal-like tumors seem to arise from luminal progenitors in which genes initiating a differentiated luminal cell fate are repressed by other mechanisms than promoter methylation, such as the Polycomb repressive complex 2 (PRC2).

Finally, epigenetic therapy, including the use of demethylating agents and histone deacetylase inhibitors, is now in clinical trials for myelodysplastic syndrome, leukemia, and ovarian and lung cancers [21]. Recent studies have suggested that co-treatment of DNA-methylation inhibitors and histone deceatylases might be an effective form of epigenetic therapy for breast cancer, as the interplay observed between DNA methylation and histone modifications can result in synergistic induction of tumor-suppressor genes $[21,44]$. Thus, a possibility exists that epigenetic therapy could play an important role in the immediate future of breast cancer treatment. The information on subtype-specific methylation profiles, described in the present study, might promote a better understanding of the epigenetic regulation mechanisms in breast cancer, thereby contributing to the improvement of epigenetic therapy.

\section{Conclusions}

In this study, we used a microarray approach followed by substantial technical and biologic validation to identify specific epigenotypes for particular subtypes in breast cancer. We clearly demonstrate the differences in the methylation profiles of basal-like and HER2-overexpressing tumors and provide evidence to support the utilization of this biomarker for prognostication and therapeutic stratification of patients with breast cancer.

\section{Additional material}

Additional file 1: Table S1. PCR primer and sequencing primer sequences and PCR conditions.

Additional file 2: Table S2. Genes differently methylated that provided optimal distinction between tumor and normal samples $(P<0.001$ and $\Delta$ $\mathrm{b}>|0.20|)$.

Additional file 3: Table S3. Comparison of quantitative methylation measurements at five CpG loci by both GoldenGate array and pyrosequencing.

Additional file 4: Table S4. Methylation levels in tumors and adjacent tissues.

\section{Abbreviations}

ANOVA: analysis of variance; CpG: cytosine-phosphate-guanine; ER: estrogen receptor; ERBB2+: ER-, PR-, and HER2+ tumor subtype; FFPE: formalin fixed, paraffin embedded; FDR: false discovery rate; GEO: Gene Expression Omnibus (GEO); HER2: human epidermal growth factor 2; hESCs: human embryonic stem cells; MLR: multivariable logistic regression; PR: progesterone receptor.

\section{Acknowledgements}

This work was supported by grants from project CGL2008-01131 (Departamento de Sanidad del Gobierno Vasco), S-PE08UN45 and PE09BF02 (Departamento de Ciencia y Tecnologia del Gobierno Vasco), BIO2008-04212, and RD06/0020/1019 (Red Tematica de Investigacion Cooperativa en Cancer, RTICC) from the MICINN. The CIBER de Enfermedades Raras is an initiative of the ISCIII. NGB had a doctoral fellowship from the Basque Government (Departamento de Educacion, Universidades e Investigacion).

\section{Author details}

'BIOMICs Research Group, Centro de Investigacion y Estudios Avanzados 'Lucio Lascaray', University of the Basque Country UPV/EHU, Miguel de Unamuno 3,1006, Vitoria-Gazteiz, Spain. ${ }^{2}$ Oral Medicine and Pathology Unit, Faculty of Medicine and Dentistry, University of the Basque Country UPV/EH, Barrio Sarriena s/n. E-48940 Leioa, Vizcaya, Spain. ${ }^{3}$ Service of Anatomic Pathology, Hospital Txagorritxu, C/Jose Achotegui s/n, E-01009 VitoriaGasteiz, Alava, Spain. " Service of Anatomic Pathology, Hospital Donostia, Paseo Beguiristain 107-115, E-20014 San Sebastian, Guipuzcoa, Spain. ${ }^{5}$ Service of Anatomic Pathology, Instituto Oncologico, C/Aldakoenea 44, E-20012 San Sebastian, Spain. ${ }^{6}$ Department of Bioinformatics and Genomics, Centro de Investigación Principe Felipe, Avda. Autopista del Saler, 164, E-6012 Valencia, Spain. ${ }^{7}$ Molecular Biology and Biochemistry, Faculty of Pharmacy, University of the Basque Country UPV/EHU, Paseo de la Universidad 7, E-01006 Vitoria-Gasteiz, Spain. ${ }^{8}$ Cancer Epigenetics Laboratory, Instituto Universitario de Oncologia del Principado de Asturias (IUOPA), HUCA, Universidad de Oviedo, E- 33006 Oviedo, Spain. ' ${ }^{\circ}$ Liverpool CR-UK Cancer Research Centre, University of Liverpool Cancer, School of Cancer Studies, 200 London Road, Liverpool L3 9TA, UK. ${ }^{10}$ Department of Immunology and Oncology, National Center for Biotechnology, CNB-CSIC, Cantoblanco, E-28049 Madrid, Spain.

\section{Authors' contributions}

$N G B, T L, A F F$, and AAS carried out the methylation assays and helped prepare the manuscript. IG, $A V, R R$, and IR provided tissues and clinical data. MCA and MJA carried out HER2, CH5/6, CK14, and EGFR1 assays and provided pathologic supports for tissue microdissection. DM and JD performed the statistical analysis. MMP, TL, MFF, MR, and JKF conceived the study and prepared the manuscript. All authors read and approved the final manuscript.

\section{Competing interests}

The authors declare that they have no competing interests.

Received: 22 April 2010 Revised: 22 July 2010

Accepted: 29 September 2010 Published: 29 September 2010

\section{References}

1. Perou CM, Sorlie T, Eisen MB, van de Rijn M, Jeffrey SS, Rees CA, Pollack JR, Ross DT, Johnsen H, Akslen LA, Fluge O, Pergamenschikov A, Williams $C$, Zhu SX, Lønning PE, Børresen-Dale AL, Brown PO, Botstein D: Molecular portraits of human breast tumours. Nature 2000, 406:747-752.

2. Sorlie T, Perou CM, Tibshirani R, Aas T, Geisler S, Johnsen H, Hastie T, Eisen MB, van de Rijn M, Jeffrey SS, Thorsen T, Quist H, Matese JC, Brown PO, Botstein D, Eystein Lønning P, Børresen-Dale AL: Gene expression patterns of breast carcinomas distinguish tumor subclasses with clinical implications. Proc Natl Acad Sci USA 2001, 98:10869-10874.

3. Parker JS, Mullins M, Cheang MC, Leung S, Voduc D, Vickery T, Davies $S$, Fauron C, He X, Hu Z, Quackenbush JF, Stijleman IJ, Palazzo J, Marron JS, Nobel AB, Mardis E, Nielsen TO, Ellis MJ, Perou CM, Bernard PS: Supervised risk predictor of breast cancer based on intrinsic subtypes. $J$ Clin Oncol 2009, 27:1160-1167. 
4. Cheang MC, Chia SK, Voduc D, Gao D, Leung S, Snider J, Watson M, Davies S, Bernard PS, Parker JS, Perou CM, Ellis MJ, Nielsen TO: Ki67 index, HER2 status, and prognosis of patients with luminal B breast cancer. J Natl Cancer Inst 2009, 101:736-750.

5. Mulero-Navarro S, Esteller M: Epigenetic biomarkers for human cancer: the time is now. Crit Rev Oncol Hematol 2008, 68:1-11.

6. Feinberg AP, Ohlsson R, Henikoff S: The epigenetic progenitor origin of human cancer. Nat Rev Genet 2006, 7:21-33.

7. Fraga MF, Esteller M: Towards the human cancer epigenome: a first draft of histone modifications. Cell Cycle 2005, 4:1377-1381.

8. Yuan J, Luo RZ, Fujii S, Wang L, Hu W, Andreeff M, Pan Y, Kadota M, Oshimura M, Sahin AA, Issa JP, Bast RC Jr, Yu Y: Aberrant methylation and silencing of $A R H I$, an imprinted tumor suppressor gene in which the function is lost in breast cancers. Cancer Res 2003, 63:4174-4180.

9. Fujii S, Luo RZ, Yuan J, Kadota M, Oshimura M, Dent SR, Kondo Y, Issa JP, Bast RC Jr, Yu Y: Reactivation of the silenced and imprinted alleles of $\mathrm{ARHI}$ is associated with increased histone $\mathrm{H} 3$ acetylation and decreased histone H3 lysine 9 methylation. Hum Mol Genet 2003, 12:1791-1800.

10. Dammann R, Yang G, Pfeifer GP: Hypermethylation of the cpG island of Ras association domain family $1 \mathrm{~A}$ (RASSF1A), a putative tumor suppressor gene from the 3p21.3 locus, occurs in a large percentage of human breast cancers. Cancer Res 2001, 61:3105-3109.

11. Krop IE, Sgroi D, Porter DA, Lunetta KL, LeVangie R, Seth P, Kaelin CM, Rhei E, Bosenberg M, Schnitt S, Marks JR, Pagon Z, Belina D, Razumovic J, Polyak K: HIN-1, a putative cytokine highly expressed in normal but not cancerous mammary epithelial cells. Proc Natl Acad Sci USA 2001, 98:9796-9801.

12. Fackler MJ, McVeigh M, Evron E, Garrett E, Mehrotra J, Polyak K, Sukumar S, Argani P: DNA methylation of RASSF1A, HIN-1, RAR-beta, cyclin D2 and twist in in situ and invasive lobular breast carcinoma. Int J Cancer 2003, 107:970-975.

13. Murata H, Khattar NH, Kang Y, Gu L, Li GM: Genetic and epigenetic modification of mismatch repair genes $\mathrm{hMSH} 2$ and $\mathrm{hMLH} 1$ in sporadic breast cancer with microsatellite instability. Oncogene 2002, 21:5696-5703.

14. Ferguson AT, Evron E, Umbricht CB, Pandita TK, Chan TA, Hermeking H, Marks JR, Lambers AR, Futreal PA, Stampfer MR, Sukumar S: High frequency of hypermethylation at the 14-3-3 sigma locus leads to gene silencing in breast cancer. Proc Natl Acad Sci USA 2000, 97:6049-6054.

15. Du Y, Carling T, Fang W, Piao Z, Sheu JC, Huang S: Hypermethylation in human cancers of the RIZ1 tumor suppressor gene, a member of a histone/protein methyltransferase superfamily. Cancer Res 2001, 61:8094-8099.

16. Di Vinci A, Perdelli L, Banelli B, Salvi S, Casciano I, Gelvi I, Allemanni G, Margallo E, Gatteschi B, Romani M: p16(INK4a) promoter methylation and protein expression in breast fibroadenoma and carcinoma. Int J Cancer 2005, 114:414-421

17. Hu XC, Loo WT, Chow LW: E-cadherin promoter methylation can regulate its expression in invasive ductal breast cancer tissue in Chinese woman. Life Sci 2002, 71:1397-1404.

18. Khan S, Kumagai T, Vora J, Bose N, Sehgal I, Koeffler PH, Bose S: PTEN promoter is methylated in a proportion of invasive breast cancers. Int $\mathrm{J}$ Cancer 2004, 112:407-410.

19. Wei M, Grushko TA, Dignam J, Hagos F, Nanda R, Sveen L, Xu J, Fackenthal J, Tretiakova M, Das S, Olopade OI: BRCA1 promoter methylation in sporadic breast cancer is associated with reduced BRCA1 copy number and chromosome 17 aneusomy. Cancer Res 2005, 65:10692-10699.

20. Orlando FA, Brown KD: Unraveling breast cancer heterogeneity through transcriptomic and epigenomic analysis. Ann Surg Oncol 2009, 16:2270-2279.

21. Feng W, Shen L, Wen S, Rosen DG, Jelinek J, Hu X, Huan S, Huang M, Liu J, Sahin AA, Hunt KK, Bast RC Jr, Shen Y, Issa JP, Yu Y: Correlation between CpG methylation profiles and hormone receptor status in breast cancers. Breast Cancer Res 2007, 9:R57.

22. Fiegl H, Millinger S, Goebel G, Muller-Holzner E, Marth C, Laird PW, Widschwendter M: Breast cancer DNA methylation profiles in cancer cells and tumor stroma: association with HER-2/neu status in primary breast cancer. Cancer Res 2006, 66:29-33.

23. Bertolo C, Guerrero D, Vicente F, Cordoba A, Esteller M, Ropero S, GuillenGrima F, Martinez-Peñuela JM, Lera JM: Differences and molecular immunohistochemical parameters in the subtypes of infiltrating ductal breast cancer. Am J Clin Pathol 2008, 130:414-424.

24. Bibikova M, Lin Z, Zhou L, Chudin E, Garcia EW, Wu B, Doucet D, Thomas NJ, Wang Y, Vollmer E, Goldmann T, Seifart C, Jiang W, Barker DL, Chee MS, Floros J, Fan JB: High-throughput DNA methylation profiling using universal bead arrays. Genome Res 2006, 16:383-393.

25. Benjamini Y, Drai D, Elmer G, Kafkafi N, Golani I: Controlling the false discovery rate in behavior genetics research. Behav Brain Res 2001, 125:279-284.

26. Gene Expression Omnibus, GEO. [http://www.ncbi.n/m.nih.gov/geo/].

27. Daskalos A, Nikolaidis G, Xinarianos G, Savvari P, Cassidy A, Zakopoulou R, Kotsinas A, Gorgoulis V, Field JK, Liloglou T: Hypomethylation of retrotransposable elements correlates with genomic instability in nonsmall cell lung cancer. Int J Cancer 2009, 124:81-87.

28. Blenkiron C, Goldstein LD, Thorne NP, Spiteri I, Chin SF, Dunning MJ, Barbosa-Morais NL, Teschendorff AE, Green AR, Ellis IO, Tavaré S, Caldas C, Miska EA: MicroRNA expression profiling of human breast cancer identifies new markers of tumor subtype. Genome Biol 2007, 8:R214.

29. Geli J, Kiss N, Karimi M, Lee JJ, Backdahl M, Ekstrom TJ, Larsson C: Global and regional $\mathrm{CpG}$ methylation in pheochromocytomas and abdominal paragangliomas: association to malignant behavior. Clin Cancer Res 2008, 14:2551-2559.

30. Konishi K, Shen L, Wang S, Meltzer SJ, Harpaz N, Issa JP: Rare CpG island methylator phenotype in ulcerative colitis-associated neoplasias. Gastroenterology 2007, 132:1254-1260.

31. Christensen BC, Marsit CJ, Houseman EA, Godleski JJ, Longacker JL, Zheng S, Yeh RF, Wrensch MR, Wiemels JL, Karagas MR, Bueno R, Sugarbaker DJ, Nelson HH, Wiencke JK, Kelsey KT: Differentiation of lung adenocarcinoma, pleural mesothelioma, and nonmalignant pulmonary tissues using DNA methylation profiles. Cancer Res 2009, 69:6315-6321.

32. Hinshelwood RA, Clark SJ: Breast cancer epigenetics: normal human mammary epithelial cells as a model system. J Mol Med 2008, 86:1315-1328.

33. Lo PK, Sukumar S: Epigenomics and breast cancer. Pharmacogenomics 2008, 9:1879-1902.

34. Pogribny IP, Beland FA: DNA hypomethylation in the origin and pathogenesis of human diseases. Cell Mol Life Sci 2009, 66:2249-2261.

35. Terada K, Okochi-Takada E, Akashi-Tanaka S, Miyamoto K, Taniyama K, Tsuda H, Asada K, Kaminishi M, Ushijima T: Association between frequent CpG island methylation and HER2 amplification in human breast cancers. Carcinogenesis 2009, 30:466-471.

36. Holm K, Hegardt C, Staaf J, Vallon-Christersson J, Jonsson G, Olsson H, Borg A, Ringnér M: Molecular subtypes of breast cancer are associated with characteristic DNA methylation patterns. Breast Cancer Res 2010, 12 R36.

37. Lim E, Vaillant F, Wu D, Forrest NC, Pal B, Hart AH, Asselin-Labat ML, Gyorki DE, Ward T, Partanen A, Feleppa F, Huschtscha LI, Thorne HJ, kConFab, Fox SB, Yan M, French JD, Brown MA, Smyth GK, Visvader JE, Lindeman GJ: Aberrant luminal progenitors as the candidate target population for basal tumor development in BRCA1 mutation carriers. Nat Med 2009, 15:907-913.

38. Carey LA, Dees EC, Sawyer L, Gatti L, Moore DT, Collichio F, Ollila DW, Sartor Cl, Graham ML, Perou CM: The triple negative paradox: primary tumor chemosensitivity of breast cancer subtypes. Clin Cancer Res 2007, 13:2329-2334.

39. Bibikova M, Chudin E, Wu B, Zhou L, Garcia EW, Liu Y, Shin S, Plaia TW, Auerbach JM, Arking DE, Gonzalez R, Crook J, Davidson B, Schulz TC, Robins A, Khanna A, Sartipy P, Hyllner J, Vanguri P, Savant-Bhonsale S, Smith AK, Chakravarti A, Maitra A, Rao M, Barker DL, Loring JF, Fan JB: Human embryonic stem cells have a unique epigenetic signature. Genome Res 2006, 16:1075-1083.

40. Bibikova M, Laurent LC, Ren B, Loring JF, Fan JB: Unraveling epigenetic regulation in embryonic stem cells. Cell Stem Cell 2008, 2:123-134.

41. Bloushtain-Qimron N, Yao J, Shipitsin M, Maruyama R, Polyak K: Epigenetic patterns of embryonic and adult stem cells. Cell Cycle 2009, 8:809-817.

42. Altun $\mathrm{G}$, Loring JF, Laurent LC: DNA methylation in embryonic stem cells. J Cell Biochem 2010, 109:1-6.

43. Calvanese V, Horrillo A, Hmadcha A, Suarez-Alvarez B, Fernandez AF, Lara E, Casado S, Menendez P, Bueno C, Garcia-Castro J, Rubio R, Lapunzina P, 
Alaminos M, Borghese L, Terstegge S, Harrison NJ, Moore HD, Brüstle O, Lopez-Larrea C, Andrews PW, Soria B, Esteller M, Fraga MF: Cancer genes hypermethylated in human embryonic stem cells. PLoS One 2008, 3 : e3294.

44. Pathiraja TN, Stearns V, Oesterreich S: Epigenetic regulation in estrogen receptor positive breast cancer-role in treatment response. J Mammary Gland Biol Neoplasia 2010, 15:35-47.

doi:10.1186/bcr2721

Cite this article as: Bediaga et al.: DNA methylation epigenotypes in breast cancer molecular subtypes. Breast Cancer Research 2010 12:R77.

Submit your next manuscript to BioMed Central and take full advantage of:

- Convenient online submission

- Thorough peer review

- No space constraints or color figure charges

- Immediate publication on acceptance

- Inclusion in PubMed, CAS, Scopus and Google Scholar

- Research which is freely available for redistribution

Submit your manuscript at www.biomedcentral.com/submit
C Biomed Central 